\title{
Ion-beam Sculpting at Nanometre Length Scales
}

\section{Citation}

Li, Jiali, Derek Stein, Ciaran McMullan, Daniel Branton, Michael J. Aziz, and Jene A.

Golovchenko. 2001. Ion-beam sculpting at nanometre length scales. Nature 412(6843): 166-169.

\section{Published Version}

http://dx.doi.org/10.1038/35084037

\section{Permanent link}

http://nrs.harvard.edu/urn-3:HUL.InstRepos:2797422

\section{Terms of Use}

This article was downloaded from Harvard University's DASH repository, and is made available under the terms and conditions applicable to Other Posted Material, as set forth at http:// nrs.harvard.edu/urn-3:HUL.InstRepos:dash.current.terms-of-use\#LAA

\section{Share Your Story}

The Harvard community has made this article openly available.

Please share how this access benefits you. Submit a story.

\section{Accessibility}




\title{
Nanoscale Ion Beam Sculpting
}

\author{
Jiali Li ${ }^{*}$, Derek Stein ${ }^{\dagger}$, Ciaran McMullan ${ }^{\ddagger}$, \\ Daniel Branton ${ }^{\ddagger}$, Michael J. Aziz ${ }^{\dagger}$, \\ and Jene Golovchenko*t
}
*Department of Physics and ${ }^{\dagger}$ Division of Engineering and Applied Sciences, Harvard University, Cambridge, MA 02138, USA
* Department of Molecular and Cellular Biology, Harvard University, Cambridge, MA 02138, USA
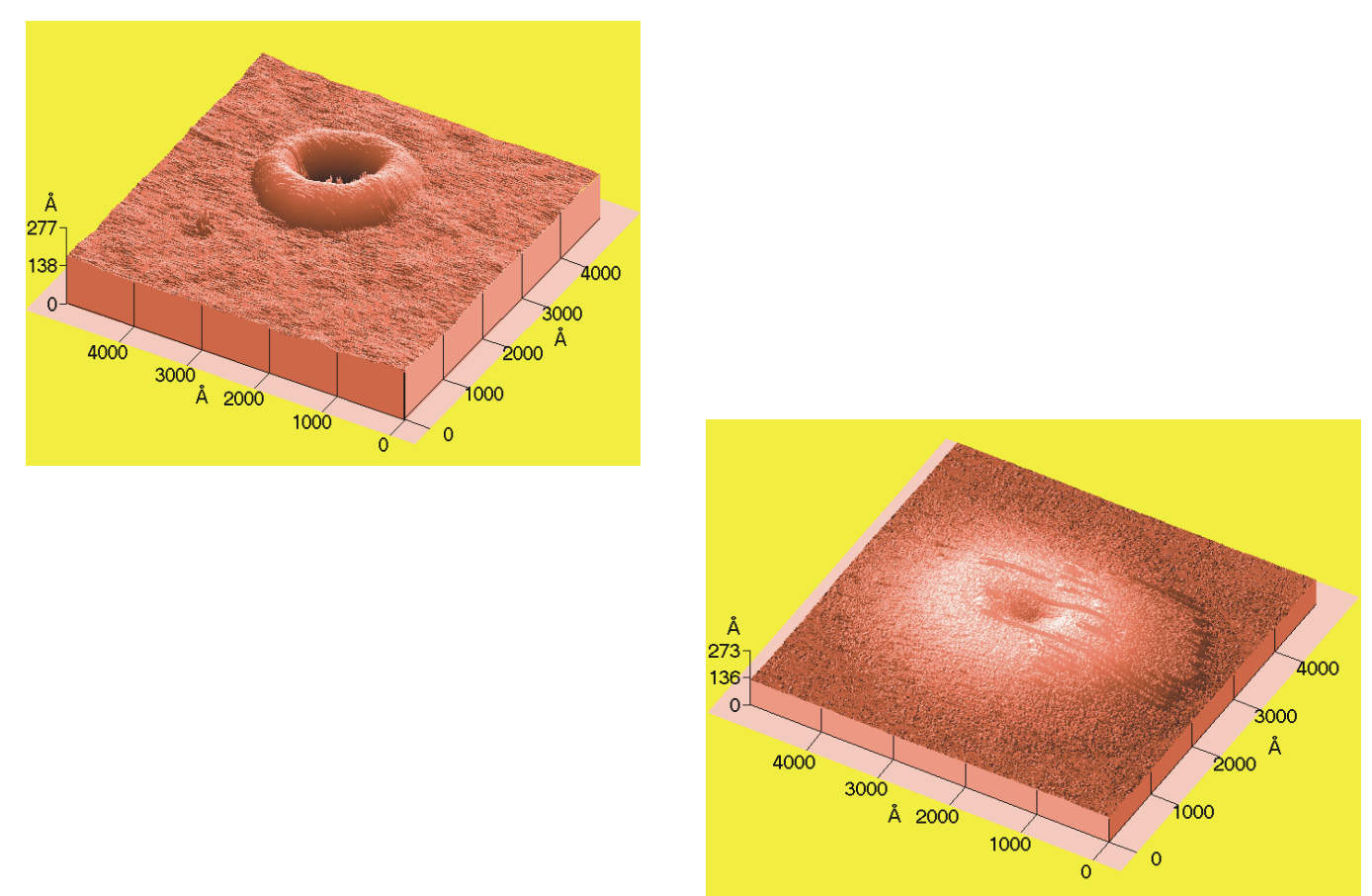
The manipulation of matter on the scale of $10^{-8}$ to $10^{-9}$ meters is an important scientific endeavor from which many future electronic, chemical and biological advances are anticipated. We report on a method to fashion materials with nanoscale dimensions that is based on a new use of low energy ion beams. The method is applied to the problem of fabricating a molecular scale hole, or nanopore, in a thin insulating solid-state membrane. We demonstrate that this new approach enables nanometer control of structural dimensions and simultaneously provides a new tool for studying ion induced materials phenomena on this length scale. We refer to the methodology as "ion beam sculpting" and present data and a physical model explaining the fundamental mechanisms that are involved. We conclude with a demonstration of a nanoscale device fashioned by the method: a molecule detector consisting of a single solid-state nanopore.

Our efforts have focused on creating single, isolated nanopores in thin insulating solid state membranes because these structures have many applications in science and technology. Such pores have been used to measure the electronic properties of single metal particles ${ }^{1}$ and molecular junctions ${ }^{2,3}$, and serve as shadow masks ${ }^{4}$ to help create other small scale

structures. Nanopores occur naturally as membrane channels in all living systems where they play a fundamental role in regulating the electronic potential and flow of ions and molecules across cellular membranes ${ }^{5}$. The dynamics and conformation of single biological nanopores with dimensions of order $2 \mathrm{~nm}$ or less can be studied electronically using patchclamp techniques ${ }^{6}$. Bio-nanopores have recently been shown to act as electronic sensors capable of identifying and characterizing single molecules in aqueous solution ${ }^{7-11}$. The incorporation of such nanoscale functionality in robust, man-made devices has far-reaching implications, but requires the manipulation of solid state materials on length scales smaller than state of the art device fabrication methods provide today.

We have used a new ion beam sculpting technique to produce 1-5 nanometer pores in thin solid state membranes designed for use as single molecule detectors of polymers like DNA, RNA and protein. The approach presented here uses silicon nitride, but the phenomena involved are not specific to this material. Samples with large ( $\sim 100 \mathrm{~nm}$ diameter) cavities or pores are fabricated first. They are then subjected to the final ion sculpting process that uses feed-back control to create structures with single digit nanometer dimensions.

\section{Experimental Procedures}

Samples for ion sculpting nanopores were prepared in thin, $500 \mathrm{~nm}$, freestanding silicon nitride membranes supported on a silicon frame. Either a bowl shaped cavity or a single initial pore of $\sim 100 \mathrm{~nm}$ diameter was created near the center of a free-standing membrane. Fig. 1a diagrams the cross-section through a membrane with a cavity, and the text accompanying the figure describes the methods used to prepare the initial cavity- or porecontaining membrane. 


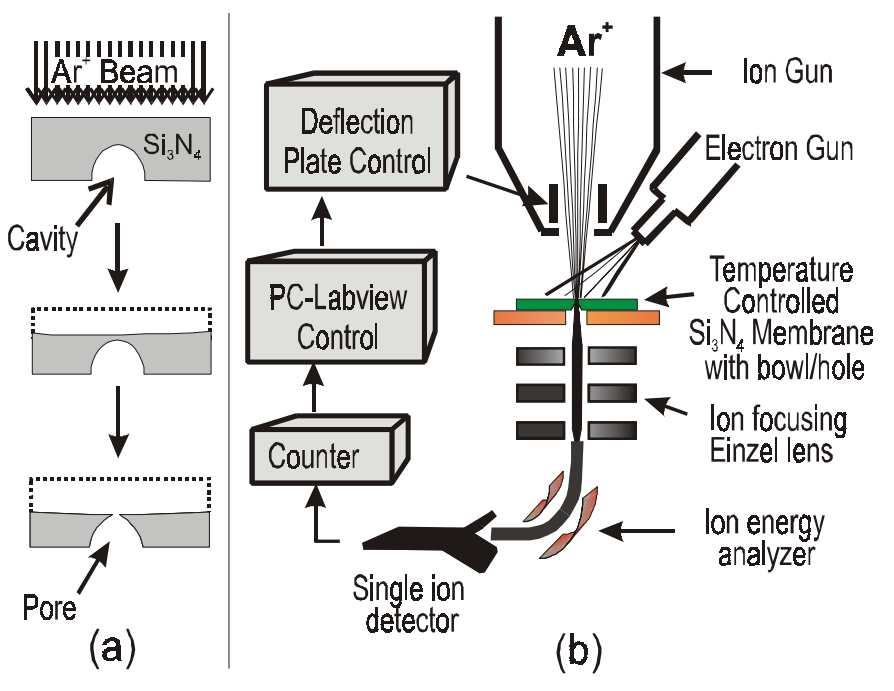

Figure 1. a) Strategy to make nanopores using argon ion beam sputtering of a free-standing $\mathrm{Si}_{3} \mathrm{~N}_{4}$ membrane with a cavity. b) Feedback-controlled ion beam sculpting system.

Sample Preparation. A Low stress ( $\sim 200 \mathrm{MPa}$ tensile) $\mathrm{Si}_{3} \mathrm{~N}_{4}$ film is deposited on a (100) silicon substrate by low pressure chemical vapor deposition $^{12}$. Photolithography and directional wet chemical etching of silicon are used to create a freestanding $25 \mu \mathrm{m} \quad \mathrm{x} 25 \mu \mathrm{m} \quad \mathrm{Si}_{3} \mathrm{~N}_{4}$ membrane ${ }^{12,13}$. Near the center of the membrane either a bowl shaped cavity (fig. 1a), or a single initial pore of

$\sim 100 \mathrm{~nm}$ diameter (not shown) is created. Initially we used a combination of e-beam lithography and reactive ion etching to create these structures in a technique developed by Ralls et al. ${ }^{14}$, but we now routinely use a Focused Ion Beam (FIB) machine ${ }^{13}$.

Ion Beam Sculpting System. The apparatus is housed in a vacuum chamber pumped to $10^{-9} \mathrm{mbar}$ by a turbo molecular pump. A differentially pumped ion gun (VG Microtech model EX05) exposes the sample's "back" flat surface to an argon ion beam of energy 0.5 to $5.0 \mathrm{KeV}$ and diameter 0.1 to $0.5 \mathrm{~mm}$ depending on the ion gun adjustments. A "Channeltron" (Gallileo Optics) electron multiplier style single ion detector is placed after the sample to count transmitted ions. A focusing Einzel lens and $60^{\circ}$ electrostatic deflection system are incorporated, between sample and detector. An electron gun (Kimball Physics Model FRA-2x1-1) floods the insulating surface of the sample with $50 \mathrm{eV}$ electrons during ion beam exposure to neutralize surface charging effects. Deflection plates at the exit port of the ion gun control ion exposure. To improve local vacuum near the sample, the sample and Einzel lens are surrounded by a liquid nitrogen cooled copper shroud. A quadrupole mass spectrometer connected to the chamber monitors the nature of the residual gases. The temperature of the sample holder was monitored with a thermocouple. and adiusted with cold $\mathrm{N}$, gas and/or a resistance heater.

Our first strategy for creating 1-5 nanometer pores in the membrane was to slowly and controllably remove material from the flat side of a membrane with a cavity on its other side (Fig. 1a, top). It is well known that when reasonably massive ions with energies of several $\mathrm{keV}$ impinge on a surface, an atomic scale erosion process, called sputtering, removes on the order of one atom from the surface for every incident ion ${ }^{15-18}$. We reasoned that as material was removed from the flat surface by this process, it would ultimately intercept the bottom of the bowl shaped cavity, forming a nanopore, as shown in Fig. 1a (bottom).

The critical issue in creating a pore only a few nanometers in diameter is to know when to stop the process. This problem is addressed by noting that immediately upon forming a pore in the cavity, the incident ions will be transmitted through the pore without impediment. Such kilovolt ions can be counted individually with better than $98 \%$ efficiency, and the rate at which they are transmitted is directly proportional to the instantaneous area of the pore. We therefore constructed an apparatus that can count the transmitted ions and use this signal to control (in this case extinguish) the incident ion beam when the desired pore size is obtained. The experimental challenge in effecting this strategy comes from the fact that the 
impinging ion beam is $10^{12}$ times more intense than the beam which passes though a $1 \mathrm{~nm}$ pore. The intense impinging beam gives rise to many sources of background noise that must be suppressed to observe the desired transmitted ion signal in the detector. The core features of the apparatus are shown in Fig. 1b, and the accompanying text describes details of its important components and capabilities. The focusing einzel lens and $60^{\circ}$ electrostatic energy analyzer are used to suppress electron, ion, and x-ray backgrounds, and are adjusted to pass the $3 \mathrm{KeV}$ argon ions used in all studies presented here. To precisely control and monitor the ion beam, electrodes at the ion gun exit port were pulsed with a 200 volt bias that deflected the ion beam off the sample with a controlled periodic duty cycle. These electrodes were also activated to permanently deflect the ion beam when final nanopore dimensions were achieved. High vacuum conditions near the sample were maintained and scrupulously monitored to minimize sample contamination from ion beam cracking of hydrocarbon vapors in the chamber.

Key parameters that influenced pore formation dynamics, and the ion sculpting process were: (1) The integrated time the ion beam was actually impinging "on" the silicon nitride membrane; (2) The sample temperature; (3) The exposure duty cycle, which we define as the explicit ratio ("on" time)/("on" time + deflected "off" time) of a single pulse cycle; and (4) the incident ion beam flux in ions $/ \mathrm{nm}^{2} / \mathrm{sec}, F$. For pulsed ion beam exposures, $F$ is the instantaneous flux when the beam is on the sample. $F$ is not an average over the pulse cycle.
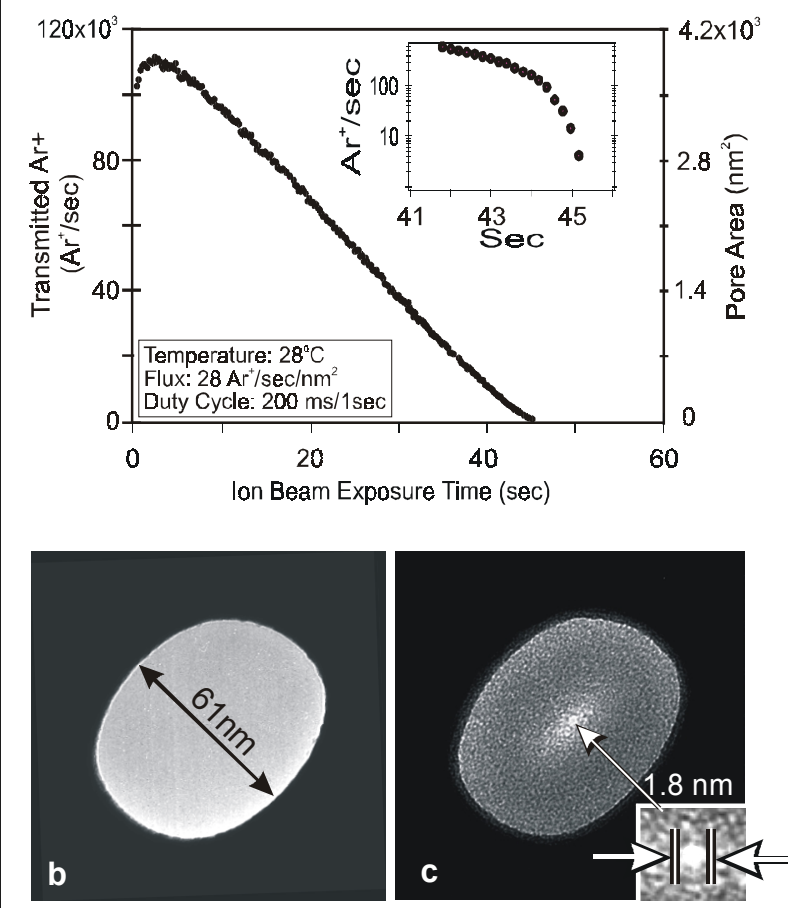

Figure 2. Sculpting a nanopore. a) Transmitted ion count rate (left axis) and pore area (right axis) vs. integrated "on" time for room temperature sample. b) TEM image of initial $61 \mathrm{~nm}$ diameter pore made by FIB in a $500 \mathrm{~nm} \mathrm{Si}_{3} \mathrm{~N}_{4}$ membrane. c) TEM image of the same sample after $\mathrm{Ar}^{+}$ion beam exposure.

\section{Experimental Results}

Surprisingly, experiments on samples with a bowl shaped cavity (as shown in Fig.1a) at room temperature did not initially yield the expected result. Nanopores did not open even after excessively long ion beam exposure. Instead, the membranes would inevitably and catastrophically fail or tear, swamping the detector with an overload of ions. The explanation for this effect was found by inserting a sample membrane in the chamber that already contained a $61 \mathrm{~nm}$ hole, formed in a focused ion beam machine (FIB) ${ }^{13}$. For such a sample held at room temperature, the counting rate clearly decreased with increasing ion beam exposure (Fig. 2a), suggesting that the pore was closing rather than opening. The incident beam was switched off when the detector counting rate fell to 40 counts/sec (Fig. 2a, inset). Transmission electron microscope (TEM) images of the pore before and after the ion beam exposure 
(Fig. $2 \mathrm{~b} \& \mathrm{c}$ ) revealed that the pore size had indeed been reduced from $60 \mathrm{~nm}$ to $1.8 \mathrm{~nm}$ !

Because the transmitted ion current is directly proportional to the area of the pore, the instantaneous pore area indicated in all our figures was calculated by multiplying the initial pore area (determined by TEM) by the ratio of the instantaneous to initial transmitted ion current. With sufficient ion beam exposure it was possible to extinguish the transmitted ion beam and completely close the nanopore as evidenced by electron microscopy.

A fundamental conclusion to be drawn from Fig. 2 is that there must be lateral atomic flow of matter along the sample surface and into the pore, in addition to the transport of matter away from the sample surface and pore region by the ion sputter erosion process. That this is a surface, or near surface, phenomenon is strongly suggested by the fact that computer simulations have shown that the ion beam energy is deposited within less than 5 nm of the sample surface ${ }^{19}$.

Although samples at room temperature with cavities never yielded nanopores under

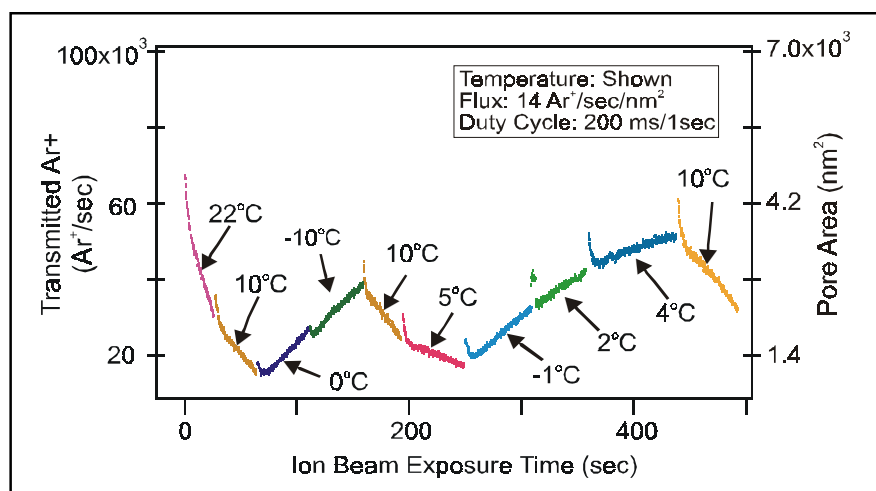

Figure 3. Ion beam sculpting, temperature dependence. the ion beam flux and duty cycle conditions of Fig. 2a, nanopores were formed when the sample temperature was reduced. Fig. 3 shows the consequence of different sample temperatures during sequential exposures of a pore. A transition between hole opening and hole closing at about $5^{\circ} \mathrm{C}$ was consistently seen under the ion beam conditions of Fig. 3. But this transition temperature also depended on ion beam parameters.

For example, with the same flux, increasing the duty cycle from $200 \mathrm{~ms} / 1 \mathrm{sec}$ to $400 \mathrm{~ms} / 1 \mathrm{sec}$, raised the transition temperature from $\sim 5^{\circ} \mathrm{C}$ to $\sim 23^{\circ} \mathrm{C}$.

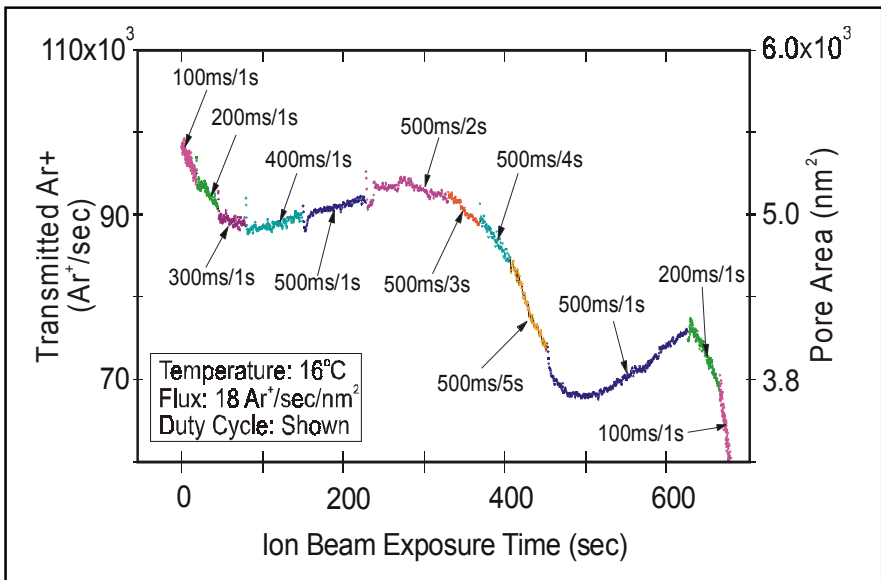

Figure 4. Ion beam sculpting, duty cycle dependence.
The duty cycle had an astonishing influence on pore opening and closing rates (Fig. 4). The pore area shrank with time under a $100 \mathrm{~ms} / 1 \mathrm{sec}$ duty cycle, but as the duty cycle was incrementally changed from $100 \mathrm{~ms} / 1 \mathrm{sec}$ to $500 \mathrm{~ms} / 1 \mathrm{sec}$, the rate of nanopore closure decelerated and by a $400 \mathrm{~ms} / 1 \mathrm{sec}$ duty cycle, the pore area actually increased. The area rate of change for $100 \mathrm{~ms} / 1 \mathrm{~s}$ and $200 \mathrm{~ms} / 1 \mathrm{~s}$ duty cycles used at the beginning and end of this experiment (Fig. 4) demonstrate the reproducibility of the data.

Other experiments revealed that the efficiency of pore closing per incident ion was clearly higher at low fluxes than at higher ones (Fig. 5). For these experiments, a continuous (rather than pulsed) exposure was used to create steady state conditions. When plotted vs. "dose" rather than "time," the slope of the data (green) revealed that the efficiency of pore 


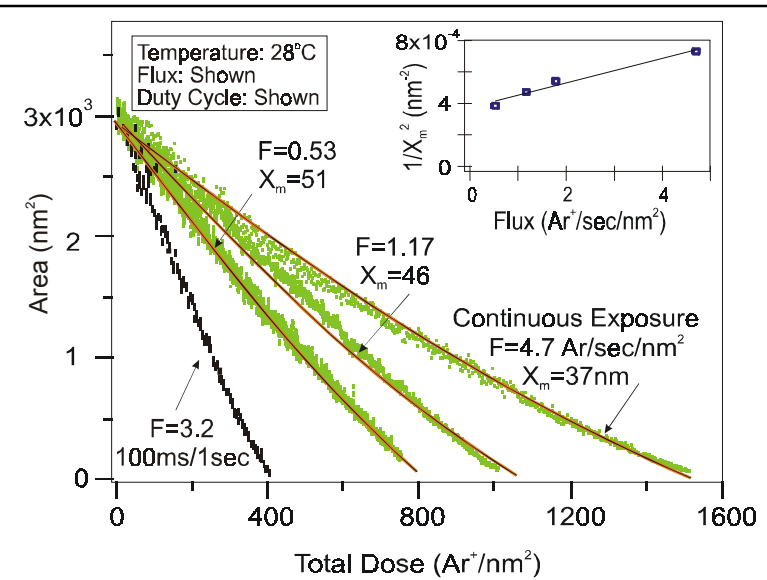

Figure 5. Ion beam sculpting, flux dependence. Pore area vs. total dose for samples exposed at different incident fluxes, $F$, to a continuous beam (green), or a pulsed beam (black). The plotted curves overlying the data points are predicted from the diffusion model (equation 1) under steady-state conditions (see text). Inset, plots $1 / X_{m}{ }^{2} v s$. flux, from which the surface diffusion constant is extracted using equation 2 . closing per incident ion was clearly higher at low fluxes than at high fluxes. For comparison, a pulsed beam experiment (black, Fig. 5) shows that the pulsed beam closed pores more efficiently than did a continuous ion beam at the same flux. As seen below, the flux dependence of pore closing efficiency provided an important clue to the fundamental processes responsible for pore closure.

Finally, to determine whether there is a limit to the pore size that can be closed, we attempted to close a series of FIB produced pores whose initial sizes ranged from $50 \mathrm{~nm}$ to 240nm. Experiments at $3 \mathrm{keV}$, $\mathrm{F}=20 \mathrm{Ar}^{+} / \mathrm{nm}^{2} / \mathrm{sec}, 28^{\circ} \mathrm{C}$, using a $200 \mathrm{~ms} / 1 \mathrm{~s}$ duty cycle demonstrated that circular pores whose diameter exceeded $\sim 160 \mathrm{~nm}$ did not close, while smaller ones did close.

\section{Model for lon Beam Sculpting}

The above experimental data are rich with empirical information applicable to creating nanopores, or other extremely small scale structures in silicon nitride. More important, the demonstrated ability to continuously monitor changing dimensions in the nanoscale range provides an unusual opportunity to test microscopic models that can account for these observations.

Overall, it seems likely that there are competing processes at work. One is responsible for opening the pore, probably driven by ion sputter erosion of the pore edge. This process likely dominates at low temperatures and high fluxes with long "on" time duty cycles. We believe that established sputtering phenomenology 17 will ultimately account for this process, although a full understanding will probably depend on knowing the detailed geometrical shape of the pore, not just its diameter.

Two different views can explain the motion of matter necessary to account for the pore closing phenomena. In the first, a very thin $(\sim 5 \mathrm{~nm})$ stressed viscous surface layer may be created by the energy deposited by the ion beam. An enhanced collective motion, driven by a reduced surface viscosity and/or enhanced surfaces stress due to implantation effects or surface tension, causes the layer to relax. Such a model has been invoked by Mayer et al. ${ }^{20}$ for atomic transport involved in sputter induced rippling phenomena in silicon dioxide. Related considerations are discussed by Carter $^{21}$ and Brongersma et al. ${ }^{22}$. (The electronic loss mechanism invoked by Brongersma et al. gives rise to pore opening rather than closing at low energies.) In an alternative approach, the transport of matter is viewed as resulting from the surface diffusion of activated atoms or molecular clusters. Both models need a driving force to account for pore closing and both need to explain the curious observed dependence of pore closing efficiency per ion on beam flux, duty cycle, and temperature. Here we describe briefly how a surface diffusion model can account for many of our 
observations. A more detailed and mathematical articulation of the ideas and results will be presented elsewhere.

The diffusion model asserts that incident ions both create and annihilate mobile species such as adatoms, ad-dimers, ad-molecules, and surface vacancies that are present in higher concentrations than in thermal equilibrium. Lacking more detailed information, it is reasonable to assume a single mobile species which, for simplicity, we will call an "adatom". It has been shown that the assumption of adatom annihilation by direct ion impingement and by diffusion to surface defects or other sinks is consistent with observations of sputterinduced ripple amplification on $\mathrm{Si}(001)^{23,24}$.

Thus we assume that the changing concentration of surface adatoms $C(\boldsymbol{r}, t)$, is governed by a two dimensional diffusion equation,

$$
\frac{\partial}{\partial t} C(\mathbf{r}, t)=F Y_{a}-F C \sigma-\frac{C}{\tau_{\text {trap }}}+D \nabla^{2} C
$$

$\boldsymbol{r}$ and $t$ are surface position and time. $D$ is the adatom surface diffusion constant and $F$ the incident ion beam flux. Aside from the diffusive component, the equation has contributions from a) a generation rate, taken proportional to the incoming flux through $Y_{a}$; b) an annihilation rate proportional to $F$ and $C$ through an annihilation cross-section $\sigma$, to account for the process in which an incident ion sputters away, or in some other way deactivates a mobile adatom from potentially filling the pore; and c) a natural annihilation rate, assumed proportional to $C$ through $1 / \tau_{\text {trap }}$, with $\tau_{\text {trap }}$ the adatom lifetime due to trapping at surface defects. The two terms involving $F$ imply that each incident ion "resets" a surface patch of area $\sigma$ to an adatom concentration $Y_{a} / \sigma$ that is independent of its previous state. It is the second such term, describing contribution (b), that allows the model to exhibit a reduction in the efficiency of pore closing per incident ion at increased fluxes.

The model assumes that the pore boundary is a "perfect sink" for adatoms, and therefore that $C$ vanishes at the pore boundary (radius $R$ ). This is the simplest boundary condition that accounts for a net accumulation of adatoms at the pore, and thus for closure. Because of its interaction with the ion beam, the pore boundary could be a net source of surface vacancies and still produce this pore-closing effect if vacancies, rather than adatoms dominate surface transport.

Solutions for the spatial adatom concentration predicted by the above model, under steady state conditions, are readily available. From them the flux of adatoms entering the pore diameter can be estimated from Fick's law. This yields a rate of pore closing dependent on the thickness and diameter of the pore. Because adatoms are being removed as well as created by the incident beam, the solutions reveal that there is an effective maximum distance from the pore edge, $X_{m}$, below which adatoms close the pore. That distance decreases for experiments performed at increasing flux. If we take the yield of adatoms per incident ion to be of order unity, and the thickness of the pore to be $10 \mathrm{~nm}$, the model yields the solid curves in Fig. 5. The single adjustable parameter required to fit the data at each flux is $X_{m}$, and these values are presented in Fig. 5 near the appropriate curve. The model predicts that

$$
\frac{1}{X_{m}^{2}}=\frac{1}{D \tau_{\text {trap }}}+\frac{\sigma}{D} F
$$

and a linear relation is indeed observed (see inset to Fig. 5). Assuming a cross section $\sim 0.1 \mathrm{~nm}^{2}$, an adatom diffusion constant of $D \sim 10^{3} \mathrm{~nm}^{2} / \mathrm{sec}$ is deduced. 
The model qualitatively explains the pulsed ion beam observations as well. When the ion beam is off, adatoms remain on the surface, but the major adatom annihilation channel associated with the incident beam flux disappears. Thus, after the beam is extinguished, the remaining adatoms may diffuse to the pore periphery at greatly increased $X_{m}$ (equation 2 with $F=0$ ). This can significantly increase the efficiency per ion for pore closing. The enhanced pore closing with increasing temperature can be accounted for by a thermally activated adatom diffusion constant. Finally the existence of a maximum size pore that can be closed under ion exposure can be explained by comparing the dependence on pore size of the effective surface area from which adatoms can be delivered to the pore to cause shrinking, relative to the size of the region at the pore periphery from which atom removal by sputtering causes the pore to open.

\section{Nanopore Detector}

Having developed a method to produce nanopores in silicon nitride membranes, preliminary investigations have been undertaken to characterize their electrical properties in aqueous salt solutions and to implement them as devices capable of detecting single molecules. Applying a voltage to electrodes immersed in a fluid compartment on each side of the membrane, an ionic current was driven through the nanopore and monitored by a high speed electrometer. This solid state analog of the single channel patch clamp technique was used to study over 50 nanopores under voltage bias and ionic strength conditions similar to those that we have used to study a lipid membrane containing an $\alpha$-hemolysin channel. The limiting pore diameter in $\alpha$-hemolysin is about $2 \mathrm{~nm}^{25}$.

The ion-sculpted material was highly resistive. Closed pores produced $20 \mathrm{G} \Omega$ seals. The total electrical resistance of the electrochemical cell was observed to be inversely proportional to the area of the nanopore as observed by TEM. Open nanopore conductances were consistent with the known conductivity of the ionic solution, assuming a pore length of $\sim 10 \mathrm{~nm}$.

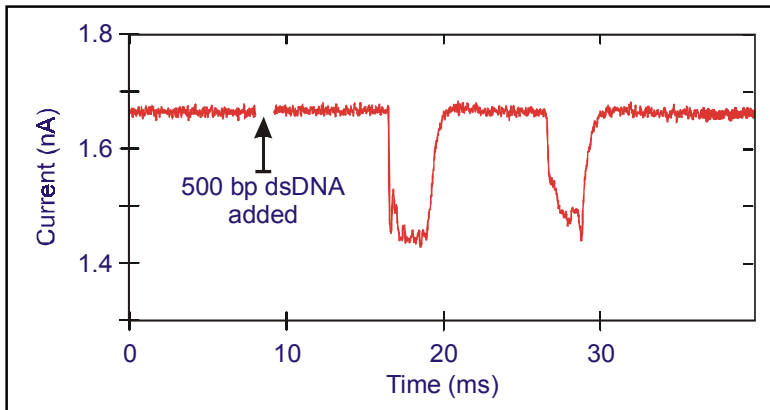

Figure 6. Molecular events in a nanopore detector.

Ion sculpted nanopores exhibitted electrical noise whose magnitude varied from sample to sample. We believe the source of the observed $1 / \mathrm{f}$ noise to be fluctuating surface charge states on the inside wall of the nanopore. Since the Debye screening length is a significant fraction of the pore diameter, it is not unreasonable that this mechanism dominates the noise spectrum. Despite our current lack of control over the electrical noise properties

of sculpted nanopores, we have observed values as low those for $\alpha$-hemolysin biopores in the quietest solid state nanopores (1.2pA rms in a $5 \mathrm{kHz}$ bandwidth).

We tested the ability of an electrically quiet $5 \mathrm{~nm}$ ion sculpted nanopore to detect double-stranded DNA molecules. Initially, with the electrochemical cell filled with only aqueous saline solution ( $1 \mathrm{M} \mathrm{KCl}, 10 \mathrm{mM}$ TRIS-HCl, 1mM EDTA, $\mathrm{pH}$ 8.0), an applied voltage of $120 \mathrm{mV}$ resulted in a constant ionic current of $1.66 \mathrm{nA}$ through the nanopore. Double stranded, 500 base-pair long, DNA was added to the negative side of the 
electrochemical cell. After allowing time for the DNA molecules to diffuse to the nanopore, we observed diminutions of the ionic current reminiscent of the blockages observed when single stranded DNA translocates through the narrower channel formed by $\alpha$-hemolysin in lipid bilayers ${ }^{8}$. Because no such blockages were seen during one hour's monitoring before adding DNA to our cell, and because the blockages ceased when the voltage bias was reversed, we attribute these blockages to the presence of DNA molecules in the vicinity of the pore. The duration of these current blockades was on the order of milliseconds, and they consistently exhibited current reductions to $88 \%$ of the open pore value. This last value is commensurate with the insertion of a rod-like molecule of cross sectional area between 3$4 \mathrm{~nm}^{2}$ in the nanopore ${ }^{26}$.

\section{Discussion and Conclusion}

The experimental observations and model considerations advance a compelling case that ion beam nanosculpting represents a promising new approach to fabricating, and fine tuning, the size of structures with extremely small dimensions. The reproducibility we have obtained does not depend on precisely matching all conditions and starting dimensions, but on the use of feed-back control to monitor the fabrication as it proceeds. Exploration of ion sculpting effects in new materials, with other ions and beam parameters, in different ambients and with other sources of surface energy, and other sources of feedback will be required to fully realize the potential of the method for creating small structures. In preliminary experiments on $\mathrm{SiO}_{2}$ we have observed ion induced atomic flows similar to those observed for $\mathrm{Si}_{3} \mathrm{~N}_{4}$, but we have not observed pore closing in crystalline silicon. Evidently the magnitude and even the sign of sculpting effects will generally depend on materials properties as well as ion beam parameters.

The diffusion based theoretical model presented above is phenomenological and contains idealizations and assumptions connected with our ignorance of many dynamical microscopic properties of matter under conditions of ion beam exposure. Nevertheless, this model accounts for all of our observations and provides estimates of these new materials parameters. Experimental and theoretical studies exploring the influence on ion sculpting of implantation effects, built-in (and ion induced) membrane stress and highly elevated temperatures, present further opportunities to refine our understanding of the ion sculpting process. It will also be important to characterize the complete atomic scale geometry, chemical activity and electronic properties of the final material that constitutes the pore region and explore ways that the surface in this region can be passivated and articulated to create reliable and sensitive nanoscale devices and sensors.

\section{Acknowledgements}

The authors wish to acknowledge financial support for this work from the U.S. Defense Advanced Projects Agency and the National Science Foundation under grants N65236-98-15407 and DMR 0073590. M.J.A. was supported by the U.S. Dept. of Energy grant DEFG02-89ER45401. This project was initiated at the Rowland Institute for Science and we acknowledge Dr. M. Burns, and W. Hill for their assistance and support during that phase of this project. In addition we thank E. Brandin, T. Denison, Dr. H. Wang, and A. Sauer for their advice and assistance. 


\section{References}

1. Ralph, D. C., Black, C. T. \& Tinkham, M. Spectroscopic measurements of discrete electronic states in single metal particles. Phys. Rev. Lett. 74, 3241-3244 (1995).

2. Reed, M. A., Zhou, C., Deshpande, M. R. \& Muller, C. J. The electrical measurement of molecular junctions. Ann. N. Y. Acad. Sci. 852, 133-144 (1998).

3. Zhou, C., Deshpande, M. R. \& Reed, M. A. Nanoscale metal/self-assembled monolayer/metal heterostructures. Appl. Phys. Lett. 71, 611-613 (1997).

4. Deshmukh, M. M., Ralph, D. C., Thomas, M. \& Silcox, J. Nanofabrication using a stencil mask. Appl. Phys. Lett. 75, 1631-1633 (1999).

5. Hille, B. Ionic Channels and Excitable Membranes (Sinauer Assoc., Sunderland MA., 1992).

6. Hammill, O. P., Marty, A., Neher, E., Sakmann, B. \& Sigworth, F. J. Improved patchclamp techniques for high-resolution current recording from cells and cell-free membrane patches. Pflugers Archiv: European J. of Physiol. 391, 85-100 (1981).

7. Bezrukov, S. M., Vodyanoy, I. \& Parsegian, V. A. Counting polymers moving through a single ion channel. Nature 370, 279-281 (1994).

8. Kasianowicz, J., Brandin, E., Branton, D. \& Deamer, D. W. Characterization of individual polynucleotide molecules using a membrane channel. Proc. Natl. Acad. Sci. U.S.A. 93, 13770-13773 (1996).

9. Akeson, M., Branton, D., Kasianowicz, J. J., Brandin, E. \& Deamer, D. W. Microsecond time-scale discrimination among polycytidylic acid, polyadenylic acid, and polyuridylic acid as homopolymers or as segments within single RNA molecules. Biophys. J. 77, 3227-3233 (1999).

10. Gu, L. Q., Braha, O., Conlan, S., Cheley, S. \& Bayley, H. Stochastic sensing of organic analytes by a pore-forming protein containing a molecular adaptor. Nature 398, 686-690 (1999).

11. Meller, A., Nivon, L., Brandin, E., Golovchenko, J. \& Branton, D. Rapid nanopore discrimination between single polynucleotide molecules. Proc. Natl. Acad. Sci. USA 97, 1079-1084 (2000).

12. Wolf, S. \& Tauber, R. N. Silicon Processing for the VLSI Era (Lattice Press, Sunset Beach Ca., 2000).

13. Stewart, D. K. \& Casey, J. D. in Handbook of Microlithography, Micromachining, and Microfabrication: (ed. Ray-Choudhury, P.) 152-195 (SPIE-International Society for Optical Engineering, 1999).

14. Ralls, K. S., Buhrman, R. A. \& Tiberio, R. C. Fabrication of thin-film metal nanobridges. Appl. Phys. Lett. 55, 2459 (1989).

15. Sigmund, P. Introduction to Sputtering. Mat. Fys. Med. Dan. Vid. Selsk. 43, 7-26 (1993).

16. Nenandovic, T., Perraillon, B., Bogdanov, Z., Djordjevic, Z. \& Milic, M. Sputtering and surface topography of oxides. Nucl. Instr. Meth. Phys. Res. B B48, 538-543 (1990).

17. Johnson, R. E. \& Shou, J. Sputtering of inorganic insulators. Mat. Fys. Medd. Dan. Vid. Selsk. 43, 403-494 (1993).

18. Gnaser, H. Ion Irradiation of Solid Surfaces (Springer Verlag, Berlin, Heidelberg, NewYork, 1999). 
19. Ziegler, J. F. \& Biersack, J. P. SRIM-2000 The Stopping and Range of Ions in Matter. http://www.research.ibm.com/ionbeams/ (2000).

20. Mayer, T. M., Chason, E. \& Howard, A. J. Roughening instability and ion-induced viscous relaxation of $\mathrm{SiO}_{2}$ surfaces. J. Appl. Phys. 76 (3), 1633-1643 (1994).

21. Carter, G. Viscoelastic buckling and plastic -flow deterministic mechanistic mechanisms for ripple initiation on ion bombarded amorphous solids. Surface and Interface Analysis 25, 952-4 (1997).

22. Brongersma, M. L., Snoeks, E., Dillen, T. v. \& Polman, A. Origin of MeV ion irradiation-induced stress changes in $\mathrm{SiO}_{2}$. J. Appl. Phys. 88, 59-64 (2000).

23. Erlebacher, J., Aziz, M. J., Chason, E., Sinclair, M. B. \& Floro, J. A. Spontaneous pattern formation on ion bombarded Si(001). Phys. Rev. Lett. 82, 2330-2333 (1999).

24. Erlebacher, J., Aziz, M. J., Chason, E. \& Aziz, M. J. Nonlinear amplitude evolution during spontaneous patterning of ion-bombarded $\mathrm{Si}(001)$. J. Vac. Sci. Technol. A 18(1), 115-120 (2000).

25. Song, L., Hobaugh, M. R., Shustak, C., Cheley, S. \& Bayley, H. Structure of ataphylococcal alpha-hemolysin, a heptameric transmembrane pore. Science 274, 18591866 (1996).

26. Bezrukov, S. M. Ion channels as molecular coulter counters to probe metabolite transport. J. Membrane Biol. 174, 1-13 (2000). 